\title{
Backward recall of noun-adjective and adjective-noun paired-associate lists
}

\author{
COLEMAN MERRYMAN, KENNETH MILLER, and GORETTI CHU \\ University of Texas at Austin, Austin, Texas 78712
}

\begin{abstract}
Subjects learned either a noun-adjective or an adjective-noun paired-associate list to a criterion of one perfect trial (Experiment I) or for three trials (Experiment II). They were then tested on all the pairs in the backward direction (Experiment I) or on half in the forward and half in the backward direction (Experiment II). The greater backward performance on the noun-adjective pairs (Experiment I) and the invariance of the noun-adjective superiority with testing direction (Experiment II) were consistent with the hypothesis of superior storage of noun-adjective pairs.
\end{abstract}

Paivio and his associates (Paivio, 1969) have shown a greater effect of stimulus than of response imagery/concreteness $(\mathrm{I} / \mathrm{C})$ on paired-associate learning. This differential sensitivity to $\mathrm{I} / \mathrm{C}$ manipulations is nicely explained by Lambert and Paivio's (1956) conceptual peg hypothesis, which states that the stimulus serves as a peg on which the response is hung and from which it can be retrieved. Both Paivio (1969) and Lockhart (1969) have hypothesized that a high-imagery stimulus provides a better retrieval cue than does a low-imagery stimulus. Alternatively, storage itself could be superior for pairs with high-imagery stimuli. Each of these two interpretations (one emphasizing storage, the other, retrieval) is consistent with the conceptual peg hypothesis.

These alternative interpretations of the conceptual peg hypothesis lead to different predictions when a test is given for backward recall. If subjects are trained to the same performance criterion on a noun-adjective ${ }^{1}$ or an adjective-noun list and then tested for backward recall, the hypothesis of superior noun-adjective storage predicts no difference in backward recall. Alternatively, if the noun-adjective superiority is due to the greater cue efficacy of the noun, then performance on the adjectivenoun list (which is cued with the noun on the backward test) should be superior.

\section{EXPERIMENT I}

\section{Method}

Subjects. Thirty-two undergraduate students, naive to verbal learning experiments, served as subjects.

Materials. Twenty-four nouns were chosen from the Paivio, Yuille, and Madigan (1968) list and were randomly divided into 12 pairs. Meaningfulness, imagery, and concreteness ranged from

Experiment I represents a course project done by the second and third authors under the direction of the first. A paper based on Experiment I was presented at the meeting of the Southwestern Psychological Association, Albuquerque, 1976. Requests for reprints should be sent to Coleman T. Merryman, who is now at the Sociology Department, Flowers Hall 212, Southwest Texas State University, San Marcos, Texas 78666.
5.0 to 7.0; all words had Thorndike-Lorge frequencies of $\mathrm{A}$ and AA. One word in each pair was made into an adjective (e.g., "corn" became "corny"). Two sets of index cards were prepared, one with the adjective-noun order and one with the noun-adjective order for each pair. One pair was printed on each index card; on the back of the card, just the stimulus was printed.

Procedure. The subjects were randomly divided into two groups of 16 subjects each. One group was shown the nounadjective order and the other group, the adjective-noun order. Cards were presented at a 2 -sec rate by the study-test method. They were shuffled after each study and each test trial during the 16-sec intertrial interval. Criterion was eight correct on any one trial. When a subject reached criterion, he was shown the response and asked to recall the stimulus. This backward test was also at a 2 -sec rate.

\section{Results and Discussion}

The noun-adjective list was learned in fewer trials than was the adjective-noun list $[\mathrm{F}(1,30)=5.20$, $\mathrm{p}<.05]$. This finding is compatible with previous research (Paivio, 1969). On backward recall, subjects who learned the noun-adjective list gave more correct responses (8.75) than did those who learned the adjective-noun list $(7.00)[\mathrm{F}(1,30)=8.79, \mathrm{p}<.01]$.

The hypothesis that noun-adjective pairs are stored better than adjective-noun pairs implies that backward performance should be equal if the two lists are taken to the same criterion. The hypothesis that the typical better performance on noun-adjective pairs is due to the greater efficacy of the noun as a retrieval cue implies that backward performance on the adjective-noun pairs should be better. We obtained superior backward performance on the noun-adjective list, a result directly counter to that predicted by the hypothesis that the noun is a better retrieval cue; the finding is also incompatible with the hypothesis of superior storage of nounadjective pairs, since both sets of pairs were taken to the same criterion.

\section{EXPERIMENT II}

Since the results of Experiment I were inconclusive, the purpose of the second experiment was to compare 
adjective-noun with noun-adjective pairs on both forward and backward tests. If the typical nounadjective superiority is due to better storage of nounadjective pairs, the difference between noun-adjective pairs and adjective-noun pairs should be the same, regardless of the direction of testing. If the difference is due to the superiority of the noun as a retrieval cue, then the superiority of the noun-adjective group should be reversed when a backward recall test is given.

\section{Method}

Subjects. The subjects were 22 undergraduate students, naive to verbal learning experiments, at the University of Texas at Austin.

Materials. Six noun-adjective and six adjective-noun pairs were formed by the same rules as in Experiment I. They were shown to subjects for three study-test trials. On the third test trial, half of each kind of pair were tested in the forward and half in the backward direction. Timing was as in Experiment $\mathrm{I}$.

\section{Results and Discussion}

The mean number of correct responses for each kind of pair is shown in Table 1. An analysis of variance revealed better performance on the noun-adjective than on the adjective-noun list $[F(1,21)=4.60, p<.05]$, bet-

Table 1

Mean Number of Correct Responses for Noun-Adjective and Adjective-Noun Pairs for Backward and Forward Tests: Experiment II

\begin{tabular}{lcc}
\hline & \multicolumn{2}{c}{ Test Direction } \\
\cline { 2 - 3 } & Forward & Backward \\
\hline Noun-Adjective & 2.45 & 1.67 \\
Adjective-Noun & 1.85 & 1.22 \\
\hline
\end{tabular}

ter forward than backward performance $[\mathrm{F}(1,21)=4.87$, $\mathrm{p}<.05]$, but no interaction $[\mathrm{F}(1,21)=1.30, \mathrm{p}>.05]$.

Experiment II presents results consistent with the hypothesis that the storage of noun-adjective pairs is superior to that of adjective-noun pairs. Lockhart (1969), however, concluded from a similar experiment that the retrieval cue, rather than the order of presentation, is responsible for the greater recall of nounadjective pairs. While Lockhart did vary the presentation order, he told his subjects that each pair could be tested in either order; consequently, his results could reflect a preference for using nouns as retrieval cues, rather than the relative ease of learning noun-adjective pairs.

The present results support the hypothesis that pairs with high-I/C stimuli are better stored than are pairs with low-I/C stimuli.

\section{REFERENCES}

Lambert, W. T., \& Paivio, A. The influence of noun-adjective word order on learning. Canadian Journal of Psychology, 1956, 10, 9-12.

LOCKHART. R. J. Retrieval asymmetry in the recall of adjectives and nouns. Journal of Experimental Psychology, 1969. 79. $12-17$.

PAIvio, A. Mental imagery in associative learning and memory. Psychological Review, 1969, 76, 241-263.

Paivio, A., Yuille, J. C., \& Madigan, S. A. Concreteness, imagery, and meaningfulness values for 925 nouns. Journal of Experimental Psychology, 1968, 76(1, Part 2).

\section{NOTE}

1. Lockhart (1969) found that abstract nouns behaved like adjectives, so the present experiment attempted to manipulate I/C by manipulating part of speech.

(Received for publication July 11, 1976.) 